\title{
Théologiques
}

Théologiques

\section{La laïcité : une façon de vivre ensemble}

\section{Micheline Milot}

Volume 6, numéro 1, mars 1998

Autres regards sur la laïcité

URI : https://id.erudit.org/iderudit/024952ar

DOI : https://doi.org/10.7202/024952ar

Aller au sommaire du numéro

Éditeur(s)

Faculté de théologie de l'Université de Montréal

ISSN

1188-7109 (imprimé)

1492-1413 (numérique)

Découvrir la revue

\section{Citer cet article}

Milot, M. (1998). La laïcité : une façon de vivre ensemble. Théologiques, 6(1), 9-28. https://doi.org/10.7202/024952ar

\section{Résumé de l'article}

La laïcité émerge, dans l'histoire des idées, comme une conception particulière de la relation entre le politique et le religieux. Toutefois, la laïcité est généralement associée à l'expérience française, et plus particulièrement à une idéologie anticléricale. Dans une acception aussi réduite, la laïcité trouve peu de pertinence pour inspirer les réflexions contemporaines sur la place et le rôle de la religion dans la sphère publique. Il nous semble important de décanter l'idée de laïcité des reliquats historiques ou idéologiques par lesquels elle peut être tenue à l'écart des débats actuels. Cette clarification nous permettra d'évaluer dans quelle mesure le concept peut être appliqué au contexte québécois, notamment en ce qui concerne les enjeux de la religion à l'école. 
Théologiques 6/1 (1998) 9-28.

\title{
La laïcité : une façon de vivre ensemble
}

\author{
Micheline MILOT \\ Département de sociologie \\ Université du Québec à Montréal
}

\section{RÉSUMÉ}

La laïcité émerge, dans l'histoire des idées, comme une conception particulière de la relation entre le politique et le religieux. Toutefois, la laïcité est généralement associée à l'expérience française, et plus particulièrement à une idéologie anticléricale. Dans une acception aussi réduite, la laïcité trouve peu de pertinence pour inspirer les réflexions contemporaines sur la place et le rôle de la religion dans la sphère publique. Il nous semble important de décanter l'idée de laïcité des reliquats historiques ou idéologiques par lesquels elle peut être tenue à l'écart des débats actuels. Cette clarification nous permettra d'évaluer dans quelle mesure le concept peut être appliqué au contexte québécois, notamment en ce qui concerne les enjeux de la religion à l'école.

The laicity has emerged, in the history of ideas, as a particular conception of the relation between politics and religion. However, the laicity is generally associated with the French experience, and in particular with an anti-clerical ideology. With reduced respect, the laicity found little pertinence to inspire contemporary reflection on the role and place of religion in the public sphere. It seems important to elucidate the idea of the laicity as historical or ideological remainders by which they could be put aside from the actual debates. This clarification will permit us to evaluate how the concept could be applied to the Quebec context, and notably with respect to the questions of religion in the schools.

Dans les divers contextes nationaux contemporains, les rapports entre l'État et la société civile comportent un mode de gestion des relations entre le politique et le religieux. Différents modèles d'équilibre entre la sphère politique et la sphère religieuse ont été réalisés, cette régulation du statut de la religion dans chaque société s'étant généralement définie, à prime abord, à l'égard de la religion dominante du pays. Toutefois, les nouvelles formes que revêt le religieux bouleversent les équilibres élaborés historiquement entre le domaine temporel et le domaine spirituel. La 
place et le rôle de la religion dans la vie sociale font l'objet d'un questionnement dans la plupart des sociétés occidentales. Dans ce contexte, la laïcité, en tant que l'un des modèles d'aménagement du religieux et du politique, a été remise à l'ordre du jour des débats intellectuels et politiques, aussi bien en France, où le concept fit son apparition à la fin du XVIII 'e siècle, qu'en d'autres pays occidentaux.

Du point de vue québécois, la question de la laïcité se retrouve souvent «stigmatisée » comme une «exception française». Pourtant, la laïcité n'en traverse pas moins l'ensemble des sociétés occidentales, même si le concept ne s'est pas traduit comme tel, ni dans d'autres langues que le français, ni dans les différentes constitutions nationales (à quelques exceptions près, comme la Turquie). Les histoires nationales ne se déroulant pas en vase clos, de grands courants culturels et symboliques ont transcendé les frontières territoriales et politiques, formant en quelque sorte une matrice de la modernité qui a atteint les différentes sociétés, selon des modalités relativement semblables. Le pluralisme, l'autonomisation des consciences, l'avènement de la démocratie, l'économie de marché sont autant de facteurs qui ont profondément transformé les logiques juridiques et culturelles des sociétés occidentales, selon des variantes nationales et divers dosages locaux. Les différents pays d'Europe, confrontés à des problèmes similaires quant au reflux du religieux, à la montée de l'islam et aux enjeux concernant l'enseignement, se sentent tous concernés par les débats sur la laïcité, comme en témoignent les nombreux colloques et publications aux titres évocateurs, dont Pluralisme religieux et laïcité dans l'Union européenne (DIERKENS 1994) et Religions et laïcité dans l'Europe des douze (BEAUBÉROT 1994a).

Malgré une sécularisation des sociétés et des cultures, le débat sur la laïcité demeure généralement source d'affects et de passions. Preuve en est les discussions houleuses, tout particulièrement en France, qu'a soulevé "l'affaire du voile", qui fut un révélateur sociologique intéressant des enjeux qui se cristallisent autour de la notion de laïcité. Preuve en est également les vicissitudes conceptuelles dont souffre cette notion. La laïcité se trouve en effet souvent confondue soit avec une réalité sociologique, l'affaiblissement du sentiment religieux, soit avec une position idéologique, l'attitude antireligieuse. Certes, cette confusion donne à voir une partie de la réalité historique ou des vecteurs ayant contribué à asseoir la réalité sociologique de la laïcité. Cependant, une distinction s'impose entre les circonstances historiques de l'émergence du concept et sa "compréhension", au sens logique de ce terme.

Notre contribution à ce numéro sur la laïcité se veut tout d'abord une clarification des connotations dont est l'objet la notion de laïcité. Nous verrons que la laïcité est avant tout une opinion sur l'État, ne disant rien 
sur la religion en elle-même, si ce n'est que le spirituel est incompétent dans le domaine temporel. La laïcité renvoie ainsi à une question politique, et non à une question religieuse (ou antireligieuse). Nous ne visons pas une étude historique de l'émergence et de l'évolution de la laïcité, d'excellentes études ayant déjà été produites sur ce sujet, notamment celles de Barbier (1995), Beaubérot et al. (1994), Beaubérot (1990), Poulat (1988) et Naurois (1960). Nous voudrions plutôt décanter la notion de laïcité des circonstances de son éclosion (bien que nous aurons à y faire référence) et de certaines conceptions voisines avec lesquelles elle est parfois confondue. Ceci nous permettra, par la suite, d'évaluer dans quelle mesure la laïcité peut ou non être appliquée au contexte québécois. Par ailleurs, dans la plupart des sociétés occidentales, les enjeux du rapport entre l'État et les religions se cristallisent habituellement autour de la place dévolue à la religion dans les établissements scolaires. C'est là un révélateur sociologique des tensions qui surgissent à propos de la place des religions dans la société civile et dans les institutions publiques. Nous y accorderons donc une attention particulière.

\section{La laicité : une notion lourdement connotée}

L'étonnante polysémie dont est auréolée la notion de laïcité traduit une appréciation contradictoire des rapports qui s'établissent entre le politique et le religieux, entre la sphère publique et la société civile. Ce qui attire particulièrement l'attention, ce sont certaines récurrences associatives qui affectent négativement l'idée de laïcité. L'une des plus largement répandues établit une synonymie entre laïcité et négation de la religion, confondant ainsi le concept de laïcité avec certains épisodes du contexte historique de son émergence. La laïcité est également souvent restreinte à une de ses formes historiques, la laïcité «à la française ». Enfin, la laïcité fait également l'objet de confusion sémantique avec des concepts voisins, en particulier celui de la sécularisation. Clarifions tout d'abord cette association entre laïcité et sécularisation, puis nous reviendrons par la suite sur les acceptions négatives que génère l'idée de laïcité.

\subsection{Laïcité et sécularisation}

La laïcité est souvent ramenée ou confondue avec une catégorie sémantique voisine, celle de la sécularisation. Les deux notions expriment cependant des réalités sociologiques qui ne sont pas identiques, bien qu'elles entretiennent entre elles des relations complexes, sociales et historiques.

Quelles distinctions peut-on établir, dans ce contexte, entre laïcisation et sécularisation? Notons que la laïcité s'est d'abord présentée comme utopie politique. Elle s'est voulue fondatrice du lien social sur une appar- 
tenance politique et non communautaire. La sécularisation, quant à elle, serait davantage de l'ordre d' " un phénomène de rupture culturelle entre la société civile et les religions, marqué notamment par l'autonomie de la connaissance et de la morale " (BEAUBÉROT 1994a : 280). Elle traduit une mutation socioculturelle globale où s'opère un amenuisement du rôle institutionnel et culturel de la religion (WILLAIME 1995 : 97). Laïcité et sécularisation peuvent se rencontrer selon des dosages différents dans une même société. Ainsi, par exemple, la Turquie possède un État constitutionnellement laïque, mais la société turque n'est pas sécularisée. En Grande-Bretagne, où l'anglicanisme est reconnu en tant que religion établie, l'État ne peut être défini comme laïque, mais la société civile est largement sécularisée et la souveraineté de l'État est entière.

La «laïcisation» est associée aux tensions entre des forces sociales dont l'enjeu est le contrôle de la sphère publique (BEAUBÉROT 1994c: 12; CHAMPION 1993 : 592). La sécularisation se caractérise plutôt par la transformation conjointe et progressive de la religion et des différentes sphères de l'activité sociale (CHAMPION 1993), un processus de perte de pertinence sociale du religieux s'effectuant au niveau des tendances lourdes de la vie sociale (BEAUBÉROT 1994c), comme la loi, le savoir, la morale. La perte de références religieuses n'est pas le produit de la laïcité, mais un fait de toutes les sociétés sécularisées.

La laïcité ne se retrouve pas traduite dans tous les systèmes politiques, bien qu'un certain degré de laïcisation imprègne les différents pays dotés d'un État moderne. Ainsi, on peut observer que les deux aspects fondamentaux de la laïcité, soit la séparation État-religions et la neutralité de l'État à l'égard de toutes les religions, sont le fait de la plupart des États démocratiques (BEAUBÉROT 1994 a,c, DIERKENS 1994, WILLAIME 1993). La sécularisation traverse l'ensemble des sociétés occidentales. Les aspects qui la caractérisent, soit la différenciation des sphères sociales et l'individualisation de la religion, ne gagnent pas seulement les groupes de croyances religieuses organisés, mais tous les groupes d'opinion structurés, y compris le laïcisme militant (MARTIN 1996, 1979).

Ces quelques remarques suffisent à suggérer que la laïcité et la sécularisation ne sont pas parfaitement juxtaposables, la laïcité renvoyant à une idée politique et la sécularisation, davantage à un processus sociohistorique.

\subsection{La laïcité : une "exception française "?}

On associe le plus souvent la notion de laïcité avec le contexte historique français de son émergence: la laïcité connote alors l'anticléricalisme ou la lutte antireligieuse, au point de s'y réduire entièrement. La 
position des acteurs sociaux influence certes les évaluations en cette matière. Les révolutionnaires français ont dû combattre l'hégémonie du pouvoir de l'Église sur la société. L'Église a pu voir en effet dans l'avènement de la laïcité un refus de la religion, un combat dirigé contre elle. $\mathrm{Si}$, à ses débuts, la laïcité a affiché une attitude anticléricale ou même hostile à l'égard du catholicisme, elle n'implique pourtant pas, en soi, un mépris de la religion. Ceci dit, l'instauration de la laïcité, en tant que décision de l'État, a infligé une restriction indéniable aux prétentions globalisantes de l'Église dans la sphère publique. Toutefois, comme le souligne É. Poulat, si "le catholicisme n'est plus religion publique, il ne s'ensuit pas qu'il soit exclu de la vie publique : simplement, il doit s'y faire sa place selon les règles du droit commun, l'état général des esprits, le rapport des forces politiques et sociales" (1988: 190).

La laïcité n'exprime pas une opinion sur la religion, mais une opinion sur l'État. La notion nous renvoie à la philosophie politique, à une façon de penser et de vivre le politique. À l'origine, c'est essentiellement une conception de la neutralité de l'État et de l'enseignement qui sert à définir la laïcité, "l'État neutre entre les religions, tolérant pour tous les cultes $^{1}$ ". La neutralité n'est pas seulement l'impartialité, qui suppose que l'État traite de façon égale toutes les religions; l'État neutre ne peut aider ni gêner aucune religion. La neutralité sera une assise fondamentale de la laicité, en tant que condition essentielle de la liberté de conscience et de religion, principe déjà affirmé en France dès la Déclaration des Droits de 1789.

Mais la neutralité exige une autre condition, essentielle à son déploiement, soit l'indépendance de l'État à l'égard des organisations religieuses. Ainsi, rapidement la laïcité se définit-elle par deux aspects complémentaires, la neutralité et la séparation ${ }^{2}$. La laïcité est le fait d'un «État neutre entre les cultes, indépendant de tous les clergés, dégagé de toute conception théologique ". La liberté de l'État à l'égard de toute religion a aussi son pendant inverse, plus rarement évoqué : les religions sont libres à l'égard de l'État. La souveraineté temporelle de l'État va de pair avec la pleine liberté religieuse. Ainsi, si la neutralité ne concerne que l'État, la séparation implique la réciprocité entre l'Église et l'État.

L'Église catholique et l'État français deviennent mutuellement exclusifs, mais par étapes progressives : d'abord par la Déclaration des Droits

1 Notamment dans le Dictionnaire de Littré, où la notion apparaît pour la première fois en 1877 .

2 Cette addition à la définition de la laïcité se retrouve, dès 1887 , dans le Dictionnaire de pédagogie et d'instruction primaire, par Fernand BUISSON. 
de 1789 , puis par la loi de séparation de 1905, enfin par la constitution, en 1946 et $1958^{3}$. La laïcité, c'est d'abord pour l'État l'assise de sa souveraineté dans le domaine temporel. Mais c'est en même temps une révolution culturelle qui transforme les mentalités et légitime de nouvelles valeurs, la liberté et l'égalité (BARBIER 1995, POULAT 1988, TOURAINE 1997). Cette façon de penser le politique se répercute sur la gestion des institutions et sur les régulations collectives de la vie sociale.

Ces deux aspects, la neutralité et la séparation, permettent de cerner la réalité politique de la laïcité, que le concept soit ou non affirmé explicitement dans le cadre juridique d'un pays. Même si le paradigme français apparaît le plus explicite, une grande variété de situations historiques et politiques correspondent, à divers degrés, à une telle conception. Le cas américain est éloquent à cet égard. Dès leur fondation, les États-Unis ont affirmé, constitutionnellement, les deux aspects de la laïcité, sans utiliser ni le terme ni un équivalent anglais. C'est la théorie du mur (wall) entre l'État et les religions. Le contexte de la fondation des États-Unis a favorisé une telle exclusion sans conflit, les colons britanniques et européens fuyaient leur pays pour trouver sur les terres d'Amérique la liberté religieuse (entre autres choses), liberté que les hommes politiques ont voulu préserver par la constitution. L'article 6 de la Constitution américaine exprime l'indépendance de l'État: "Aucune déclaration religieuse spéciale ne sera jamais requise comme condition d'aptitude aux fonctions ou charges publiques sous l'autorité des États-Unis». La neutralité est stipulée dans le premier amendement: "Le Congrès ne pourra faire aucune loi concernant l'établissement d'une religion ou interdisant son libre exercice ». Aux États-Unis, comme en France, ni la loi ni la constitution ne président à la "création" de la laïcité : c'est bien davantage un état d'esprit, une conception prévalante dans les consciences qui se trouvent cristallisés dans les lois et la constitution.

La laïcité, dans sa version à la française, "s'affirmait comme une utopie politique capable de réaliser un État où le lien politique est à la base du lien social "(RÉMY 1993 : 366). Ce qui signifie que l'appartenance s'est traduite par un lien politique entre le citoyen et l'État, et non par la médiation des appartenances à une catégorie sociale, un groupe ou une église. La construction de la citoyenneté suppose que soient reléguées au second rang les appartenances communautaires, dont l'inflation risque toujours de mettre en péril le lien politique. La laïcité fut un projet de société et la proposition d'une certaine "spiritualité " républicaine, à

3 Pour un exposé historique des combats épiques qui eurent lieu entre l'Église et l'État pendant presque deux siècles en France, on consultera les ouvrages de Poulat, Barbier, Beaubérot ou Naurois, que l'on retrouve en bibliographie. 
l'encontre des prétentions globalisantes de la spiritualité catholique : une sorte de religion civile à la française, appuyée sur l'intention de socialisation à une commune appartenance à la nation, sur une morale de citoyen et sur un civisme qui traduirait l'adhésion à la République, bref un dispositif mental d'entrée dans la démocratie laïque.

\subsection{Laïcité et anticléricalisme}

Il faut reconnaître qu'en France, au regard de la séparation à l'américaine qui se fit sans conflit à la faveur d'un contexte politique et social nouveau, la laïcité de l'État n'a pu se définir qu'en s'opposant aux prétentions globalisantes de l'Église catholique sur la société civile qu'elle investissait à l'époque de part en part. Son pouvoir temporel s'étendait au droit (contrôle juridictionnel sur les actes des souverains par le pouvoir d'excommunication), à la politique (octroi de la légitimité du pouvoir dans la mesure où il lui est assujetti), à l'économie (doctrine sociale concernant le travail, le prêt à intérêts, la charité, le salaire, etc.), aux mœurs (vie familiale, vie sexuelle, etc.), au contrôle des opinions (appréciation exclusive de l'orthodoxie) et des savoirs "scientifiques» (contrôle de la diffusion des connaissances, censure, prétention intellectuelle pour évaluer des énoncés scientifiques à partir de critères dogmatiques). La collision frontale entre les prétentions laïques et catholiques s'avérait inévitable. Même si l'impulsion fondamentale visant la séparation du temporel et du spirituel était d'abord anti-dogmatique, le camp laïque s'est trouvé pris dans une logique symétrique, bien qu'inversée, à celle du camp ecclésial: il ne pouvait s'affirmer qu'en niant le pouvoir catholique, versant ainsi dans l'anticléricalisme ${ }^{4}$.

La laïcité émerge d'une matrice catholique, mais elle s'en est dégagée jusqu'à se retourner contre elle pour se légitimer, ce qui fait dire à Jean Rémy que "dans un tel contexte, la laïcité prouve sa pugnacité dans son opposition à un adversaire. L'anticléricalisme devient un ciment pour l'action, avec le risque que la laïcité s'organise elle-même comme un contre-cléricalisme séculier, avec une contre-prétention monopolistique " (1993 : 368). Les circonstances historiques particulières à la France expliquent en grande partie ces tensions. Ni la laïcité ni l'Église n'avaient encore renoncé à leurs prétentions monopolistiques et globalisantes sur la société civile. C'est ce qui a encouragé une perception de la laïcité comme étant confondue avec un dogmatisme antireligieux concurrent de la religion. La recherche laïque d'une unité fondatrice du lien social peut,

4 Le problème s'est posé différemment dans les pays protestants, le protestantisme présentant une certaine décléricalisation interne et externe de l'Église. 
en se radicalisant, se définir par la négation de la diversité, et donc se refermer sur une logique dogmatique, la laïcité recréant alors ce qu'elle proscrit.

De cette époque d'affrontement, on relève plus facilement les radicalités du camp läque que les intransigeances de l'Église catholique et de certains milieux religieux, qui s'opposèrent jusqu'à la constitutionnalisation de la laicité, en 1946 et $1954^{5}$. Les pressions émanant de la société civile ne furent cependant pas les seules qui contribuèrent à faire céder la digue du rapport à l'autorité ecclésiale. À l'intérieur même du christianisme, la Réforme, en affirmant la préséance du libre examen, avait façonné une conception individualiste en matière de conviction. On ne peut donc s'en tenir à une opposition simple entre clercs et laïcs pour résumer la nature de la laïcité et de ses effets sur le catholicisme.

Bien que les circonstances d'émergence de la laïcité comportent indéniablement une opposition aux prétentions temporelles de l'Église, la laïcité a, depuis ce temps, opéré elle-même la critique de son idéologie issue des Lumières. À vouloir faire le procès d'une laïcité suspectée de parti pris idéologique antireligieux, on risque de défoncer des portes ouvertes. Il faut davantage retenir que la laïcité consiste d'abord et avant tout en une façon de penser et de vivre le politique, qui garantit les conditions de la liberté religieuse et de l'égalité. Elle est à la fois une éthique et une méthode de pensée.

\section{L'exclusion fondatrice : "L'Église libre dans l'État libre "}

Autonome à l'égard des religions, l'État ne privilégie ni ne subventionne aucun culte ${ }^{6}$. Autonomes vis-à-vis de l'État, les religions peuvent se développer librement dans la société civile, sous la seule réserve de l'ordre public. "La laïcité exprime juridiquement la liberté même de l'acte de foi..." (BARBIER 1995: 78). Le principe de la liberté religieuse fait aujourd'hui partie du droit commun des pays occidentaux qui ont un État moderne. Le contenu de cette liberté religieuse est précisé par l'affirmation que nul ne doit être privilégié ou pénalisé en raison de ses croyances. C'est bien la liberté de conscience et de religion qui est

5 Émile POULAT a produit l'une des études les plus documentées sur ces questions dans Liberté, laïcité. La guerre des deux France et le principe de la modernité. Paris, Cerf/Cujas, 1988.

6 Ce qui n'empêche pas plusieurs États de financer les Églises, comme diverses associations, pour des activités à caractère social ou culturel. Par exemple, un financement peut être accordé pour la construction de mosquées, dans la mesure où la mosquée tient aussi lieu de centre culturel ou communautaire. 
reconnue publiquement à tout individu, non la religion en tant qu'Église et pouvoir.

La laïcité se décline avec les acquis des droits de l'homme. Plus précisément, selon Émile Poulat, l'idéologie des droits de l'homme s'est constituée en rupture avec les principes du catholicisme de l'époque (1988: 49). Autrement dit, il a fallu sortir de la logique religieuse de l'État pour accéder à la conception démocratique des droits. Les droits de l'homme s'avèrent le vecteur essentiel d'une conception libérale de la société et du monde. Alain Touraine, dans son dernier livre au titre évocateur, Pourrons-nous viure ensemble? Égaux et différents, revient avec cette idée que la laïcité, en tant que séparation de l'Église et de l'État, est un élément essentiel de la démocratie :

Si l'idée de laïcité signifie la séparation de l'État et des Églises et le respect par la loi de toutes les croyances et pratiques religieuses qui sont en conformité avec les principes du droit, elle appartient à la définition même de la modernité. Si l'appel à la laïcité est une manière de s'opposer à l'intolérance d'États qui n'acceptent pas la pratique de religions ou d'opinions minoritaires, je me proclame laique militant et sans concessions. Je comprends même que l'esprit laïque soit resté associé à l'anticléricalisme ou au rejet actif de toute religion dans les pays où une Église a longtemps été associée à un pouvoir autoritaire qui en appelait à la religion dominante pour réprimer ceux qui n'y appartenaient pas " (1997: 332). Pour Touraine, le principe de laîcité s'oppose à la théocratie autant qu'à la religion civile ${ }^{7}$.

Si la séparation entre l'État et la religion permet à chacune de ces deux instances une autonomie accrue, c'est qu'elle suppose une séparation plus fondamentale entre l'État et la société civile. La modernité politique implique précisément une distinction entre l'État et les intérêts particuliers des individus, l'État se préoccupant de l'intérêt général de la communauté politique, les individus pouvant évoluer dans la société civile en toute liberté. Cette distinction est d'ailleurs corrélative à l'apparition des droits politiques (ceux du citoyen) et des droits de l'homme (droits civils de l'homme comme individu). Ainsi se constituent une sphère publique,

7 Ainsi pour Alain Touraine, le principe de laïcité condamne autant la Constitution civile du clergé imposée par la France révolutionnaire que l'identification de certains théologiens de la libération d'Amérique centrale avec un pouvoir politique (1997: 312-313). Roger Mehl (1983) soutient la même idée, jusqu’à déclarer la loi de séparation de 1905 d'antilaïque, en ce qu'elle maintient un contrôle de type gallican sur l'Église et lèse gravement la liberté ecclésiale. 
domaine de l'État et des droits des citoyens, et une société civile, domaine des libertés individuelles, où la religion a toute liberté.

La séparation entre État et religion, entre sphère publique et société civile, s'est opérée en France dans un climat conflictuel où l'Église se percevait comme victime d'un acte d'hostilité. Comme nous l'évoquions plus haut, ce contexte historique a probablement nourri la confusion regrettable à l'effet que la disjonction entre le politique et le religieux confine la religion à la vie privée, c'est-à-dire qu'elle ne disposerait plus d'aucune légitimité pour intervenir dans la vie sociale. Selon Maurice Barbier, ce raisonnement est inexact. Affirmer la séparation de l'Église et de l'État n'équivaut aucunement à interdire le rôle social de la religion. Il faut distinguer la sphère publique, qui renvoie au domaine de l'État, et la sphère privée, à celui de la société civile. "Dire que la religion relève de la sphère privée ne signifie pas que c'est seulement une affaire privée et personnelle. Cela signifie qu'elle échappe au domaine public de l'État, mais qu'elle peut exister et agir librement dans la société. La religion n'est donc pas réduite à sa dimension intérieure et individuelle; elle a nécessairement un caractère extérieur et social " (BARBIER 1995: 85). La laïcité écarte la religion de l'État, mais lui laisse entière liberté dans la société. C'est en ce sens qu'Émile Poulat parle de sociétés sorties du christianisme, quelle que soit la place et l'influence que celui-ci y conserve (POULAT 1995 : 410).

Ces remarques permettent de nuancer l'opposition entre sphère privée et sphère publique. Quand on affirme que, dans les sociétés modernes, la religion est relayée au privé, il ne faut pas entendre que la liberté religieuse est confinée au for intérieur de chacun. Les libertés de culte, d'expression et d'association ne peuvent se déployer uniquement dans l'intimité du foyer. Elles s'exercent nécessairement dans l'espace social, dans la société civile. Ainsi, la laïcité "a un lien évident avec la liberté religieuse, car elle lui permet d'exister et lui apporte même la garantie de l'État "(BARBIER 1995: 79). On a confondu à tort les sociétés athées avec la laïcité, ce qui est une erreur théorique, fondée sur une prémisse fausse. Les régimes stalinien et hitlérien ont bien sûr démontré une hostilité féroce à toute idéologie religieuse ou fondée sur une vision transcendante. Dans ces cas, l'État tend à absorber entièrement la société civile, selon la logique de non séparation entre les deux domaines. Cette négation ne servit en fait qu'à se positionner en radicalisant la logique qu'ils pourchassaient, c'est-à-dire dans un dogmatisme étouffant et totalitaire.

L'État laïque, en se reconnaissant incompétent dans le domaine du spirituel et n'intervenant pas sur ce terrain, assure néanmoins le cadre juridique garantissant la liberté religieuse et l'exercice des cultes. La séparation de l'État d'avec l'Église a délié la société civile de toute emprise 
totalisante, ce qui a peut-être encouragé une évolution de la religiosité vers une conception moins régulée, plus individualiste et délibérative.

\section{3. Évolution de la laïcité : au-delà du silence de César...}

\subsection{Les enjeux éthiques}

La notion de laïcité et sa réalité sociologique sont évolutives. La laïcité-abstention à l'américaine, ou la laịcité-neutralité à la française, semblent de plus en plus des positions appelées à évoluer face à la complexité des enjeux de la société civile. D'une part, la reconfiguration idéologique de l'espace social, sous la poussée du pluralisme, soulève des questions face aux revendications à fondement religieux. La religion n'est pas que l'affaire des Églises ou des associations religieuses. Quel que soit le statut juridique de la confessionnalité dans la constitution de l'État, la religion n'échappe pas au droit et est, par conséquent, affaire d'État. Ce regard obligé du politique ne concerne pas seulement le mode de gestion du principe de séparation État-religions. D'une part, la pression de l'opinion publique oblige les pouvoirs étatiques à prendre en compte les différents aspects de l'expression individuelle des croyances qui se traduisent dans des pratiques qui affectent le domaine public. D'autre part, sous la pression des nouveaux enjeux éthiques dans lesquels interviennent les associations religieuses, l'État ne peut se contenter d'être un régulateur neutre entre des propositions éthiques plurielles, voire divergentes. Tout en veillant à ce que l'autonomie des personnes et la liberté de conscience soient respectées, l'État se voit placé en position de devoir assurer des règles morales génératrices d'un lien social, rendant possible le vivreensemble, condition permanente pour "faire société ${ }^{8}$ ". Il y a là bien sûr un terrain de choix pour que les religions tentent une percée nouvelle dans la sphère publique (sous forme de partis politiques ou autrement).

Après une étape nécessaire d'exclusion réciproque, les religions et l'État en arrivent peut-être à une reconnaissance mutuelle, chacun demeurant sur son terrain respectif. Le rôle social de la religion, s'il n'apparaît pas souhaitable pour tous, n'en est pas moins effectif, bien que dans une mesure toute relative. Poulat parle d' " une nouvelle civilité ", Barbier d'une «collaboration entre l'État et les religions». Certains espéreront davantage : "Ne serait-il pas imaginable qu'on dépasse l'attitude de la tolérance ou l'attitude d'un simple dialogue respectueux des différences, à travers lequel finalement il n'y a pas tellement de créations communes

8 Ce qui rappelle la thèse fondamentale de Durkheim, pour qui l'existence de valeurs communes est absolument nécessaire au lien social. 
ni de devenir qui transforme une réalité donnée au départ?» (MONTCONDUIT $1990: 259$ ).

Plusieurs croyants ou groupes religieux peuvent y voir un moment favorable pour réinvestir la société et la culture. L'État n'est pas sans ignorer l'influence que les organisations religieuses peuvent avoir sur leurs fidèles, et que les exigences morales ou civiques qu'elles énoncent peuvent être en contradiction avec celles qu'il met de l'avant. En outre, étant donné que les institutions politiques ont perdu la confiance d'une grande partie des citoyens, plus encore que les institutions religieuses, il n'est pas impossible que les valeurs holistiques et diffuses de la religion apparaissent légitimes et exercent un certain attrait. Enfin, on observe le reflux sur la scène sociale des intégrismes religieux issus des trois traditions monothéistes (KEPEL 1989) qui proposent des théories englobantes du politique et du social, sur la base de contre-valeurs de la modernité. Cette nouvelle donne dans la configuration idéologique de l'espace social ne peut qu'interpeller la sphère publique, qui relève de l'État. Toutefois, l'État libéral s'interdit de limiter l'action des Églises, pourvu qu'elles n'outrepassent pas les principes du droit et le maintien de l'ordre social. Dans les faits, on observe en général un très grand libéralisme dans le traitement réservé par chaque État aux organisations religieuses, les seules limitations ou restrictions se réfèrent à la moralité, à l'hygiène et à l'ordre publics, aux libertés des individus, à la légalité fiscale et financière et au droit au travail sans discrimination, notamment celle liée au genre.

\section{2 "Il n'y a pas de maître du sens "}

Le problème politique de l'État ne concerne pas en tant que tel la pluralité des options religieuses endossées par les citoyens. Il en va plutôt des effets de cette diversité : les religions ou les associations à références religieuses seront-elles capables de se rencontrer dans un espace social commun, ou chercheront-elles à refermer le sentiment d'appartenance de leurs fidèles sur la base d'un communautarisme concurrentiel à références religieuses?

Comment concilier le pluralisme des opinions, donc leur contradiction absolue, $/ \ldots /$ avec le maintien d'une vérité? Est-ce que démocratie signifie ipso facto relativisme intellectuel : à chacun son opinion et, une fois ce principe posé, tâchons de trouver l'accord sur des décisions pratiques qui réalisent le meilleur compromis entre les exigences de chacun. Ou est-ce que, au-delà du pluralisme, nous pouvons maintenir l'idée de vérité en l'associant à ce qui est la règle du jeu démocratique, la contradiction et le conflit? À l'évidence, la conviction religieuse représente un cas privilégié de cette interrogation (GAUCHET $1990: 254-255$ ). 
Il est possible d'affirmer que les sociétés modernes sont laïques en tant qu'elles affichent une conception non dogmatique des valeurs. "Il n'y a pas de maître du sens ", de dire Marcel Gauchet. Mais les nouvelles donnes qui surgissent sur la scène sociale appellent à un contenu positif de la laïcité, autrement dit, on ne peut s'en tenir au "silence de César". La laïcité ne renvoie plus à une idéologie politique combative, se définissant par le mode de l'opposition au cléricalisme, mais comme un dispositif instituant un espace de construction de la citoyenneté. Malgré les points de vue divergents, entre ceux qui pensent que la laïcité a perdu de sa vigueur et de sa légitimation et ceux qui croient qu'elle n'a pas une ride, elle devient surtout la condition d'un pouvoir-vivre et d'un vouloir-vivre ensemble (TOURAINE 1997). "Elle offre ainsi la base acceptable par tous d'un contrat social renégocié dans une société divisée mais attachée à ses libertés " (POULAT $1988:$ 198).

Bien sûr, on pourra toujours objecter que dans l'application, les choses ne sont pas aussi nettes. En effet, ce qui vient d'être dit n'empêche pas que les aménagements soient plutôt mixtes en ce domaine, chaque État tissant des relations diverses avec les religions, dominantes principalement, concernant différents secteurs de l'activité sociale. Par ailleurs, les Églises ne sont pas sans tenter parfois de jouer de leur influence dans la sphère publique, notamment en matière d'éducation et d'enjeux éthiques, comme l'avortement ou l'euthanasie. En outre, si le pluralisme religieux fait l'objet d'une reconnaissance sociale et politique, il ne découle pas de cette reconnaissance une égalité de droits, les religions dominantes ou historiques continuant de bénéficier de privilèges particuliers, de natures institutionnelle et financière. Enfin, le danger demeure qu'à trop valoriser les particularismes, on fragmente la société, empêchant qu'une appartenance commune puisse se déployer suffisamment pour garantir le lien social nécessaire au vivre-ensemble en société.

Les enjeux qui entourent les rapports entre religions et école dans nos sociétés illustrent ces préoccupations, puisque l'institution scolaire est un lieu de convergence des attentes et des intérêts sociaux à l'égard des nouvelles générations. Nous aborderons ces enjeux à partir du terrain québécois, tout en reconnaissant qu'ils se posent de façon relativement similaire dans les autres contextes nationaux occidentaux. Nous nous y référerons, au besoin, pour éclairer la problématique québécoise.

\section{Le Québec et la laïcité : les enjeux de la transmission scolaire}

À partir de ce panorama rapidement esquissé des caractéristiques de laïcité, nous pouvons nous demander dans quelle mesure le Québec peut ou non être considéré laïque. La société québécoise est largement sécula- 
risée, d'une part. Mais d'autre part, si l'on reprend les deux aspects fondamentaux qui définissent la laïcité, soit la séparation de l'État et des religions et sa neutralité à l'égard de toutes les religions, le Québec n'est pas laïque. Cette double absence se traduit principalement en matière d'éducation publique. Les deux confessions religieuses historiques font partie de la structure de l'État jusqu'à ses plus hauts niveaux, soit le Conseil supérieur de l'Éducation, où l'on retrouve un comité catholique et un comité protestant. Les structures scolaires des deux principales villes, Montréal et Québec, sont confessionnelles, catholiques et protestantes, sous la protection de la Constitution canadienne ${ }^{9}$. La quasi totalité des écoles publiques sont confessionnelles, catholiques ou protestantes. Enfin, l'enseignement religieux confessionnel de ces deux traditions est garanti légalement dans toutes les écoles; les parents ont cependant le choix entre cet enseignement et un enseignement moral.

Dans le jeu de la dynamique sociale qui sous-tend le processus de sécularisation, "la religion peut se modifier ou restreindre ses prétentions de manière à légirimer (ou même contribuer à provoquer) une certaine perte de son emprise, ce qui lui permet, paradoxalement mais logiquement, de garder un statut privilégié et une influence diffuse » (BEAUBÉROT 1994a: 282). C'est ce que l'on a pu observer en matière d'éducation au Québec. Les écoles confessionnelles ne présentent pas un climat endoctrinant ou prosélyte marqué; les enseignements religieux, tout en demeurant sous le contrôle des comités confessionnels, ont atténué passablement leurs objectifs typiquement confessionnels ou catéchétiques. Il y a donc une certaine dissonance, théorique et juridique, au regard de la réalité sociologique sécularisée et pluraliste. Plusieurs citoyens s'accommodent implicitement d'une situation politique et institutionnelle qui, tout en ne correspondant pas à la réalité sociologique, ne semble pas perturber celle-ci. La "religion scolaire" s'accorde même, par des procédés d'atténuation, d'ajustement et d'adaptation, aux logiques de la modernité, notamment l'individualisme religieux et les quêtes diversifiées relatives au sens. Dans un tel contexte, la réflexion sur la laïcité at-elle une pertinence?

9 Au moment d'écrire cet article, une demande de la part du gouvernement du Québec a été déposée de manière à ce que ces protections confessionnelles garanties par la Loi constitutionnelle de 1867 du Canada ne s'appliquent plus au Québec. Le débat est transposé à Ottawa où s'exprime une féroce opposition de la part de la droite catholique et protestante. 


\subsection{Religions et socialisation scolaire}

Quand on aborde la problématique des rapports entre religion et école, on s'aperçoit vite que des questions fondamentales s'y trouvent impliquées, devenant ainsi un excellent révélateur sociologique. Les transactions politiques établies entre l'État et les religions, le rôle reconnu à ces dernières dans la sphère publique et la configuration religieuse prévalant dans la société détermineront les rapports entre monde scolaire et institutions religieuses. Ainsi, quel que soit le contexte national, l'école fut toujours le centre de débats houleux et est encore l'objet d'intenses pressions concernant la question religieuse.

Généralement, au centre des débats, on retrouve les demandes multiples provenant de divers groupes religieux (prescriptions alimentaires, vestimentaires, exigences de non-mixité, etc.), l'enseignement religieux et les requêtes institutionnelles (écoles séparées, financement public, enseignement religieux particulier). Ces requêtes se fondent sur le principe de réciprocité, eu égard aux privilèges dont jouissent généralement les religions dominantes. Les divers modes sociétaux de gestion du sens se traduisent par un aménagement qui doit assurer une éducation pour tous, selon des objectifs de socialisation commune, tout en respectant les libertés fondamentales : celle de croire, comme celle de ne pas être importuné par les convictions d'autrui. L'enseignement des cultures religieuses et des valeurs qu'elles portent prend, dans une telle problématique, une dimension particulière.

Les régimes de séparation entre l'État et les religions accusent une grande diversité, mais les libertés religieuses sont reconnues dans tous les États modernes. Cependant, sur une telle prémisse, on peut s'opposer à ce que la religion joue un rôle à l'école, comme on peut également revendiquer qu'elle ait une place dans l'éducation. Au nom de la liberté de conscience et de pensée de chaque enfant, les partisans d'une laïcité plaideront pour une école neutre sur le plan religieux, seule garantie de l'égalité et de la liberté de chacun. Au nom de la liberté publique d'enseignement, qui est une liberté associative, d'autres appuieront l'existence d'écoles et d'enseignements religieux confessionnels.

Une gestion pragmatique de ces positions contradictoires peut aller dans deux directions : soit assurer une neutralité confessionnelle des établissements publics, soit garantir à toutes les dénominations religieuses, comme aux visions séculières de l'existence, un droit à la gestion d'une éducation conforme à leurs valeurs respectives. On le pressent : au nom de l'égalité et de la liberté, les occasions de conflits sont multiples.

Cependant, on observe que, malgré des régimes de séparation ÉgliseÉtat dans la plupart des pays occidentaux, les traditions dominantes histo- 
riquement bénéficient généralement de privilèges particuliers, et ce même dans des régimes de laïcité comme la France. La séparation peut ne pas exclure une certaine délégation de responsabilités, à une ou plusieurs Églises, s'accompagnant d'une aide financière directe ou indirecte. Ainsi tout citoyen contribue, sans s'en rendre compte, au financement de certains privilèges accordés à certaines religions. Le principe d'égalité et de neutralité de l'État est ainsi atteint, et peut faire l'objet de contestations juridiques, comme on le voit dans la plupart des pays qui adoptent cette position modérée, contestations qui proviennent généralement de groupes religieux minoritaires revendiquant des aménagements similaires.

\subsection{L'école laïque est-elle antireligieuse?}

Les conceptions antinomiques autour de l'école sur la question religieuse ne traduisent plus une lutte autour du savoir, mais un conflit dans l'appréciation des normes qui doivent façonner les citoyens dès leur socialisation scolaire. L'école doit-elle prendre en considération les besoins religieux des élèves? Quels principes doivent la guider? Jusqu'où peut-elle aller, dans la reconnaissance comme dans l'indifférence?

Jules Ferry, l'un des penseurs influents de la laïcité en France, considéré comme le "père de l'école laïque ", confia un jour à son collègue Jaurès que son but était bien "d'organiser l'humanité sans Dieu". Cependant, il adopta une position beaucoup plus libérale à l'égard de l'école, qu'il institua "neutre», et non pas " antireligieuse ". La circulaire qu'il fit parvenir aux instituteurs est restée célèbre, par sa précaution éthique: "Parlez avec la plus grande réserve, dès que vous risquez d'effleurer un sentiment religieux dont vous n'êtes pas juge ". Il voulut faire de l'école un lieu de rencontre se situant au-delà des différences, en considérant que si l'institution scolaire ne pouvait qu'être laïque, les enfants, eux, ne l'étaient pas nécessairement.

Habilement, Jules Ferry s'appuyait sur une thèse "recevable " pour la diplomatie pontificale de l'époque (même si elle fut l'objet de combats houleux avec les milieux catholiques français), en affirmant que « l'école doit être läque, au nom de ce principe conforme à la tradition : une barrière infranchissable entre le temporel et le spirituel ». Cette légitimation, que l'on retrouve dans le testament de Ferry (1890) renvoie en effet aux positions du Vatican, qui à l'époque, au nom de sa propre autonomie à l'égard du gallicanisme, validait cette séparation, même si le Saint-Siège n'était pas prêt à l'assumer jusque dans ses conséquences ultimes.

La laïcité de l'éducation en France a contribué à constituer "l'espace scolaire laïque comme lieu d'accueil universel» (RÉMOND 1990: 163). Elle représente donc une organisation d'accueil pour une société reconfi- 
gurée idéologiquement par le pluralisme des options religieuses et philosophiques. Elle structure une sorte de "construction de l'espace de la citoyenneté ", elle est instituante d'un espace public commun, en mettant au premier plan ce qui est semblable entre les élèves. L'appartenance citoyenne, sans nier les appartenances sociales ou religieuses, est passée au premier plan. Selon Jacqueline Costa-Lascoux ${ }^{10}$, l'école publique «a aidé à la formation d'une citoyenneté universaliste, fondée sur une forte séparation entre l'appartenance citoyenne et les appartenances sociales ou religieuses $\gg(1996: 82)$, école et citoyenneté devenant intimement associées dans l'école républicaine. L'école s'inscrit alors comme le lieu qui génère la proximité, nécessairement au prix d'une occultation institutionnelle des catégorisations religieuses ou philosophiques qui sont porteuses d'inégalités et d'évaluation. L'école devient alors le lieu de circulation de la différence, le lieu instituant la pluralité comme une valeur. Toutefois, la laïcité n'impose pas aux élèves la neutralité (COSTA. LASCOUX 1996: 72). C'est à partir de ce principe que les signes vestimentaires ont finalement été reconnus comme acceptables dans l'école républicaine.

Alain Touraine ira plus loin, en se demandant si la "noble" idée de laïcité, là où elle n'a plus à combattre un pouvoir totalitaire religieux, ne va pas trop loin en retirant du système scolaire toute information sur la religion. Selon l'auteur, l'" enseignement des religions, de leurs croyances comme de leur histoire, n'est assurément pas une atteinte à la laïcité; c'est au contraire le silence imposé sur ces réalités religieuses qui est une atteinte inacceptable à l'esprit d'objectivité et de vérité dont se réclame l'école laïque et qui ne doit pas davantage être absent d'écoles dites religieuses, qu'il faut protéger plus directement encore contre l'enfermement communautaire " (TOURAINE 1997 : 334).

\subsection{L'harmonisation entre religion, école et société civile}

$\mathrm{Si}$ les formes juridiques qui balisent les rapports entre religion et école ne sont pas toutes laïques, les consciences individuelles le sont dans leurs interactions dans la société civile, ce qui fait dire à Émile Poulat que "nous sommes tous laïques" $(1988: 191)$. Les trajectoires des individus ne sont plus déterminées par un héritage culturel ou religieux, elles s'individualisent. La réflexion sur la laïcité s'inscrit dans un processus historique général des sociétés modernes qui recherchent des repères éthiques et culturels hors des religions. En ce sens, la laïcisation est

10 Jacqueline Costa-Lascoux a animé pendant trois ans, dans le cadre d'une Commission nationale en France, un groupe de réflexion L'expression religieuse dans une société laïque. Voir également sa publication sur la laïcité, en bibliographie. 
inévitable. Elle ne constitue pas une menace pour la religion. Elle peut, au contraire, ouvrir un espace de délibération favorable à une prise en compte des faits religieux en cadre scolaire, en harmonisant cette reconnaissance aux droits fondamentaux et aux valeurs de la société pluraliste. L'identité commune des citoyens provenant d'horizons différents ne peut cependant pas se légitimer sur un passé commun. Ainsi, bien que certaines traditions religieuses constituent un élément important du patrimoine et de la culture d'un pays, on ne peut en appeler de la nécessité de la mémoire de ce passé religieux pour restructurer une identité commune, passablement anémique aux yeux de certains. Cet héritage religieux en tant que mémoire collective ne représente plus une justification valable en contexte pluraliste.

Les expériences et les enjeux religieux qui sont présents dans la société civile invitent à une réflexion en profondeur sur les principes qui peuvent guider les rapports entre religion et école. Cependant, comme le souligne Jean-Paul Willaime qui a étudié les rapports entre religions et écoles dans différents contextes nationaux, "la situation de l'enseignement concernant la religion apparaît particulièrement complexe : problèmes des objectifs de cet enseignement, de son contenu, de son contrôle par l'État et/ou les autorités religieuses, de son intégration dans un projet éducatif global" (WILLAIME 1993: 393). Trois aspects de la question nous semblent devoir être considérés dans la recherche d'une harmonisation entre la société séculière, où évoluent une pluralité de visons du monde, et la sphère publique, espace commun des citoyens dans leur rapport avec l'État. L'État ne peut faire obstacle à la libre interrogation du sens par les hommes, qui relève d'un droit fondamental admis dans toutes les sociétés occidentales. Toutefois, comme nous l'avons exposé plus haut, cette interrogation ne se confine pas au for intérieur; elle s'exprime à travers un certain nombre de pratiques ou de préférences morales. Elle nécessite donc un régime qui permette la mise en œuvre de la réalisation de ce sens. Toutefois, cette expression ne saurait se déployer au détriment d'un dispositif qui, lui, favorise le développement d'un sentiment d'appartenance à l'État, par l'éducation à la citoyenneté.

Ceci n'a rien d'évident pour certaines sensibilités religieuses ni pour certaines sensibilités lä̈ques, dans la mesure où les unes et les autres n'ont pas renoncé à leur prétention universelle. Les religions, comme dans le cas de l'islam et de certains milieux catholiques ou protestants, ne cachent pas leur désir de réinvestir la société civile sur la base d'une fonction sociale articulée aux déficits de la modernité, notamment à la vacuité du sens et à l'anomie généralisée. N'est-ce pas la propension naturelle de toute idéologie religieuse que d'informer tout le social, de voir l'unique et l'uniforme comme garant de l'unité? Par ailleurs, une vision 
laïque peut être tentée de se positionner en symétrie par rapport à ces poussées religieuses. Dans un cas comme dans l'autre, la notion d'unité n'est pas loin de se confondre avec celle de l'uniformité, excluant la différence et privilégiant l'assimilation de l'autre ou son rejet.

Il importe d'engager une réflexion en profondeur sur les principes de philosophie politique qui sont l'essence de l'idée de laïcité. La laïcité, débarrassée des reliquats idéologiques anticléricaux et du modèle français républicain, fait corps avec la démocratie qui garantit la liberté pour chacun de croire ou de ne pas croire. Cela suppose pour les uns de laïciser leur conception sur la laïcité et, pour d'autres, de se dégager d'une vision étriquée et passéiste d'une laïcité à la française.

\section{Bibliographie}

BARBIER, Maurice. 1995. La laïcité. Paris, Éditions L'Harmattan.

BEAUBÉROT, Jean, dir. 1994a. Religions et laïcité dans l'Europe des douze. Paris, Syros.

BEAUBÉROT, Jean et al. 1994b. Histoire de la laicité. CRDP de FrancheComté, CNDP.

BEAUBÉROT, Jean. 1994c. "Laïcité, laïcisation, sécularisation " dans Pluralisme religieux et laïcité dans l'Union européenne. (DIERKENS, A. éd.) Bruxelles, Éditions de l'Université de Bruxelles, pp. 9-25.

BEAUBÉROT, Jean.1990a. La laïcité, quel héritage? De 1789 à nos jours. Genève, Labor et Fides.

BEAUBÉROT, Jean. 1990b. Vers un nouveau pacte laïc, Paris, Seuil.

CHAMPION, F. 1993. "Les rapports Église-État dans les pays européens de tradition protestante et de tradition catholique : essai d'analyse ", Social Compass, 40, 4.

COSTA-LASCOUX, Jacqueline. 1996. Les trois âges de la laïcité. Paris, Hachette.

DIERKENS, A. éd. 1994. Pluralisme religieux et laïité dans l'Union européenne. Bruxelles, Éditions de l'Université de Bruxelles.

GAUCHET, Marcel. 1985. Le désenchantement du monde. Histoire politique de la religion. Paris, Gallimard.

GAUCHET, Marcel. 1990. "Les enjeux véritables du débat de la laïcité " dans Nouveaux enjeux de la laïcité. (RÉMOND, René, dir.) Paris, Centurion, pp. 252-255. 
HERVIEU-LÉGER, Danièle. 1996. "Le déferlement spirituel des nouveaux mouvements religieux", dans Identités religieuses en Europe. (DAVIE, G., et HERVIEU-LÉGER, D., dir.), Paris, La Découverte, pp. 269-290.

KEPEL, Gilles. 1989. La revanche de Dieu. Chrétiens, juifs et musulmans à la conquête du monde. Paris, Seuil.

MARTIN, David. 1996. "Remise en question de la théorie de la sécularisation", dans Identités religieuses en Europe. (DAVIE, G., et HERVIEU-LÉGER, D., dir.) Paris, La Découverte, pp. 25-42.

MARTIN, David. 1978. A general theory of secularisation. Oxford, Basil Blackwell.

MEHL, Roger. 1983. "La notion française de laïcité et son évolution ", Parole et société 3-4, 160-175.

MONTCONDUIT, F. 1990. "Repenser la laïcité de l'État », dans Nouveaux enjeux de la laïcité. (RÉMOND, René, dir.), Paris, Centurion, pp. 255-260.

NAUROIS, Louis de. 1990. Le fondement philosophique et le régime juridique de la laïcité en droit français. Paris, PUF.

POULAT, Émile. 1988. Liberté, Laïcité. La guerre des deux France et le principe de la modernité. Paris, Cerf/Cujas.

POULAT, Émile. 1990. "Les quatre étapes de la laïcité », dans Nouveaux enjeux de la laïcité. (RÉMOND, René, dir.), Paris, Centurion, pp. 31 . 42.

RÉMOND, René, dir. 1990. Nouveaux enjeux de la laïcité. Paris, Centurion.

RÉMY, Jean. 1993. "Laïcité et construction de l'Europe ", dans Religions et transformations de l'Europe. (VINCENT, G. ET WILLAIME, J.-P., dir.) Strasbourg, Presses Universitaires de Strasbourg, pp. 365-380.

TOURAINE, Alain. 1997. Pourrons-nous viure ensemble? Égaux et différents. Paris, Fayard.

WILLAIME, Jean-Paul. 1995. Sociologie des religions. Paris, P.U.F., (collection Que sais-je?).

WILLAIME, Jean-Paul. 1993. "Univers scolaires et religions en Europe de l'Ouest " dans Religions et transformations de l'Europe. (VINCENT, G. ET WILLAIME, J.-P., dir.), Strasbourg, Presses Universitaires de Strasbourg, pp. 381-396. 